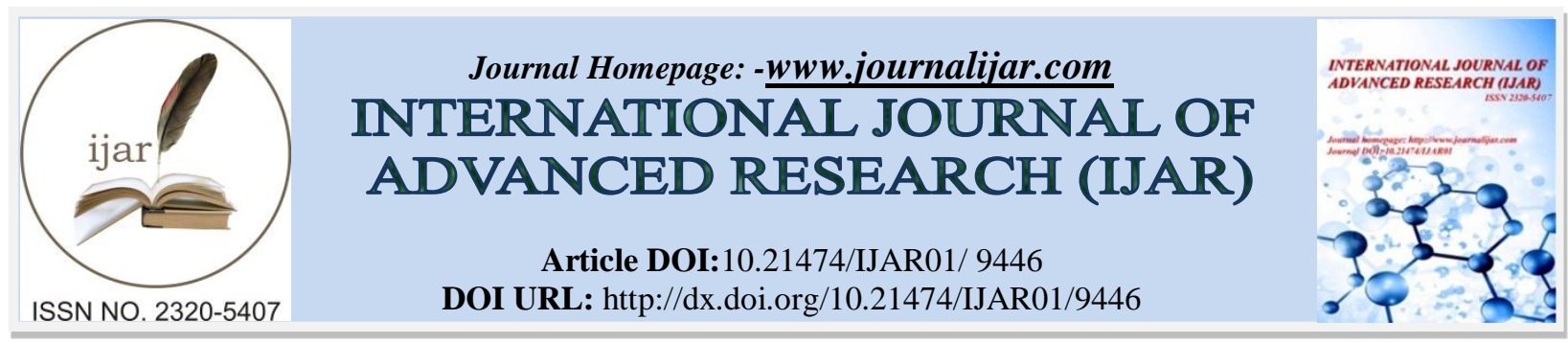

RESEARCH ARTICLE

\title{
QUANTUM PROBABILITY IN THE FRAMEWORK OF PHILOSOPHY.
}

\author{
J. Wang. \\ School of Physical science and Technology, Guangxi Normal University Guilin, 541004, P. R. China.
}

\section{Manuscript Info}

Manuscript History

Received: 24 May 2019

Final Accepted: 26 June 2019

Published: July 2019

\begin{abstract}
In wave mechanics of quantum theory, the involved wave function in the Schrödinger equation is treated as the probability of deflected particles in some spatial region, which is fundamentally different with that of the statistical mechanics in the framework of classical physics. Consequently, the understanding of the quantum probability and its relation to the definite value reported by the measurement becomes the core problem to understand the quantum measurement. In this paper, we discuss the interpretations for quantum probability from the point of view of determinism and empiricism.
\end{abstract}

Copy Right, IJAR, 2019,. All rights reserved.

\section{Introduction:- \\ Origin of Quantum Probability:-}

In spite of the success of quantum mechanics in describing the micro-world, there is no consensus about connotation of wave function. Part of the founders of quantum mechanics, e.g. Erwin Schrödinger, and De Broglie, gave the semi-classical interpretation. However, several of them tried to interpret as the real wave, in order to overcome the intrinsically logical contradiction. In 1926, Max Born, enlightened by Einstein's wave-particle duality, proposed the statistical interpretation of wave function [1,2]. According to Albert Einstein, the electromagnetic wave can be considered as light quanta, moving along some track, and the square of amplitude denotes the probability in the region of the track. Generalized to physical particles, the corresponding matter waves imply that the appearance of particles in some spatial region obeys the statistical law, instead of the causality obeyed by real waves. Accordingly, the matter waves are probabilistic waves, and the motion of corresponding particles obeys the probabilistic statistics. While the probabilistic amplitudes propagate following the law of causality. From the mathematical structure of quantum mechanics, we just can estimate the measure of some probability for the specified result. It is the discontinuity of particle structures of matter that results in the probability in quantum mechanics and causes the problem in the interpretations for quantum probability [3, 4].

\section{Nature of Quantum Probability:-}

According to the statistical interpretation of quantum probability, we can just get the probability of the observable quantity in the quantum measurement. However, in the practical experiment, we must perform measurements for many times, instead of once, and then can obtain the statistical probability. Therefore, the quantum probability denotes the statistical effects in the system formed by a great deal of identical particles prepared in the measurements. However, in classical physics, the probability satisfies the countability and additivity. Taking the double-slit experiment as an example, we first keep just the slit "S1" open and then observe the pattern as $\psi_{1}$ and the probability as $\mathrm{P}_{1}$ on the detector, while we just keep the split "S2" open and then get the pattern of $\psi_{2}$ and the probability of $\mathrm{P}_{2}$ on the detector. However, if both "S1" and "S2" are opened, then the pattern appears on the screen

Corresponding Author:-J. Wang.

Address:-School of Physical science and Technology, Guangxi Normal University Guilin, 541004, P. 
is not just the simple addition of $\psi_{1}+\psi_{2}$, but with interference pattern $2\left|\psi_{1}\right|\left|\psi_{2}\right|$. Accordingly, the essential addition is the addition of probabilistic amplitudes $P_{1}+P_{2}=\left|\psi_{1}+\psi_{2}\right|^{2}=\left|\psi_{1}\right|^{2}+\left|\psi_{2}\right|^{2}+2\left|\psi_{1}\right|\left|\psi_{2}\right|$, instead of the addition of intensity, which is the key and significant concept in quantum mechanics. There are two meanings for the quantum system. Firstly, we can understand as probability for an isolated system without measurable properties. The second and the most important is a pure state provides us the maximum information of the system. Actually, all the observable properties can be figured out from the knowledge of state function.

We just consider the probable values of observables and tread them as numbers. Then these numbers appear in the section of " 0 " and " 1 ", with a probability " $\mathrm{P}(\mathrm{E})$ ". As far as the system in a state of "S", there must be a probability in the measured sets " $E$ " of the measurement for observable " $\mathrm{m}$ ". In the double-split experiment, there are three states in the measured results, i.e. $\mathrm{W}_{1}$ resulting from the pass through "S1", $\mathrm{W}_{2}$ resulting from the pass through "S2", and the interference $\mathrm{W}_{3}$. The resulting mixed state " $\mathrm{W}=\mathrm{aW} \mathrm{W}_{1}+\mathrm{bW}_{2}+\mathrm{cW}_{3}$ " represents the probability under the given measuring condition, i.e. the set of observables including all the observables " $m$ " and all the possible values of " $m$ ", instead of the probability of each pure state. The essence of the quantum probability, in the implicative statement, denotes the conditional probability of the possible result, which includes the measurements for both events already happened and events not in place.

\section{Statistical Determinism in Quantum Probability:-}

The statistics in quantum mechanics is a kind of statistics in nature, instead of the general statistics on the ground of the causality. For example, in the experiment of radioactive decay, it is found that the number of radioactive nucleus "N(t)" decreases with time, because of its decay, which satisfies the exponential statistical law $N(t)=N(0) e^{-t / \tau}$. According to quantum mechanics, we can give appropriate theoretical illustration for such statistical law. However, there is no any theory can predict the moment of decay for the individual nucleus. Furthermore, it has been demonstrated that the decay of individual nucleus is the random behavior. The individual decay is independent and has no causal relation to the other ones. Therefore, the quantum statistics just gives the statistical distribution for the measured results of a great deal of events. The quantum statistics is based on the identical principle of particles. As a result, we cannot distinguish the particles and thus cannot find out the reason of randomness for the identical particles under identical external conditions.

The other characteristic of the statistical determinism is that the fundamental quantity in quantum mechanics is the probabilistic amplitude, instead of the probability. The probabilistic amplitude is the state function describing the state of a quantum system, which is a complex function. The square of modulus of probabilistic amplitude $|\psi|^{2}$ gives the probability. It is the state function, i.e. the probabilistic amplitude, that satisfies the state superposition principle, instead of the probability. In quantum mechanics, the only quantity that describes the evolutionary causality is the probabilistic amplitude, instead of specific physical quantity. The significance of wave function lies in the reflection for the statistical distribution of measured results for a great deal of micro-events. Consequently, it is natural that we can causally describe the individual event.

According to quantum mechanics, the measured result of dynamical variable corresponds to the one of the eigenvalues of corresponding operator $\hat{F}$. If measuring the dynamical variable for many times in the same state $\psi$, we can get the mean value $\langle\hat{F}\rangle=\frac{(\psi, \hat{F} \psi)}{(\psi, \psi)}$, in which the round bracket denotes the scalar product. After modifications, we rewrite the mean value as $\langle\hat{F}\rangle=\sum_{n} P_{n} \lambda_{n}$, where $P_{n}=\frac{\left|\varphi_{n}, \psi\right|^{2}}{(\psi, \psi)}$ is the probability corresponding to the eigenvalue $\lambda_{n}$, i.e. the frequency of the appearance of $\lambda_{n}$ in the all measured values. Such quantum probability $P_{n}$ is worked out from the theory, instead of the classically derived result on the ground of causality.

Finally, since the quantum probability is the statistical theory for the identical particles, the concept of ensemble [5, 6] can be applied to it, which is distinct, however, with that in the classical framework. In quantum mechanics, each measured result is the eigenvalue, instead of the mean value. Accordingly, it needs to realize the ensemble during the measuring process. Specifically speaking, we need to prepare the particles, which form the ensemble, under same macro-condition, in order to accumulate sufficient data to calculate the mean value and to ensure repetition of the measurement for many systems in the same state. 


\section{Ontological Interpretations for Quantum Probability:-}

In order to give a causality interpretation for the quantum probability, the wave-particle duality, and the quantum jump, it was proposed that the unbroken wholeness of the universe relates to the separated systems by the quantum interconnectedness [7], according to the hierarchy of supersystem, system, and subsystem. It is postulated that the wave function is the description for a real quantum field and that the particles move randomly and always connects to the field. The probability density is determined by the final distribution of a stationary state results from the stochastic motion [8].

In the ontological framework, the understanding of quantum probability is established in the process of quantum measurement, which is divided into two stages. The first stage is the interaction between the measured system and the measuring apparatus, both of which obey the law of quantum mechanics. When the interaction occurs, the wave function of the combined system is decomposed into the summation of non-overlapping wave-packets, and each wave packet corresponds to each measured result, i.e. each quantum channel. During the interaction, every particle enters into one channel and stay there, according to its initial state and the structure of quantum potential. From then, the influence of quantum potential on particle is only determined by the wave-packet, corresponding to the quantum channel. Therefore, the quantum probability can be understood as the probability that the particle enter into the quantum channel. The inactive wave-packet, which is not occupied by particles, still can lead to interference between the apparatus and wave-packets, which have influence on the quantum potential. Consequently, the independent and separated measured result is unstable and changeable. The second stage is that the measured result is enlarged by some apparatus and becomes the macro-phenomenon on the macro-level. Then the apparatus particle interacts with the particles in macro-system, and the quantum measurement becomes the unchangeable and irreversible process. In this sense, the quantum probability is completely determined by randomness that the particles enter into the quantum channel.

\section{Summary:-}

According to the fundamental postulates of quantum mechanics, the wave function is just a mathematical symbol and has no any physical meaning. What is really significant is the square of its modulus, which leads to the arguments on the problem of quantum probability. Quantum probability, different with the classical statistical probability just with methodological significance, is an operative concept and directly relates to the measured results, which represents the description for the properties of quantum objects on the level of ontology. The theory of quantum probability originates from the fundamental postulate and endows the solutions of Schrödinger equation with statistical property. In quantum mechanics, it is the probabilistic amplitude that satisfies the superposition principle, whose square determines the probability. The superposition of probabilistic amplitude includes an interference term, comparing with that of probability, which causes new features.

\section{References:-}

1. M. Born, "Physical Reality", in the Philosophical Quarterly, 3, 139 (1953)

2. M. Jammer, "The Philosophy of Quantum Mechanics", John Wiley \& Sons, Inc, New York, 1974

3. W. Heisenberg, "Physics and Philosophy", New York: Harper and Row, 1958

4. C. A. Hooker, "The Nature of Quantum Mechanical Reality: Einstein Versus Bohr", R. G. Cologny, ed. Paradigms and Paradoxes: The Philosophical Challenge of Quantum Domain, University of Pittsburgh Press, 1972

5. L. E. Ballentine, "The Statistical Interpretation of Quantum Mechanics", Rev. Mod. Phys., 42, 358 (1970)

6. D. Home, M. A. B. Whitaker, "Ensemble Interpretations of Quantum Mechanics: A Modern Perspective", Phys. Rept., 210, 223 (1992)

7. D. Bohm, B. J. Hiley, "On the Intuitive Understanding of Nonlocality as Implied by Quantum Theory", Foundation of Physics, 5, 93 (1975)

8. D. Bohm, B. J. Hiley, "The Undivided Universe: An ontological interpretation of quantum theory", London: Routledge and Kegan Paul, 1993. 